Escudero Escorza, T. (2019). Evaluación del profesorado como camino directo hacia la mejora de la calidad educativa. Revista de Investigación Educativa, 37(1), 15-37.

DOI: http://dx.doi.org/10.6018/rie.37.1.342521

\title{
Evaluación del profesorado como camino directo hacia la mejora de la calidad educativa
}

\author{
Teacher evaluation as a direct path towards \\ the improvement of educational quality
}

\author{
Tomás Escudero Escorza' \\ Catedrático Emérito del área de Métodos de Investigación y Diagnóstico en Educación \\ Universidad de Zaragoza
}

\begin{abstract}
Resumen
El artículo presenta y defiende la evaluación del profesorado como una faceta muy relevante de la investigación evaluativa en educación, con un gran potencial para fortalecer y mejorar diversos aspectos de la calidad educativa, si el proceso de evaluación se lleva a cabo y se contextualiza adecuadamente, siguiendo los principios y características de la evaluación defendidas en numerosas publicaciones científicas de importantes expertos nacionales e internacionales. En el artículo se fundamenta la evaluación correcta analizando aspectos importantes como los enfoques modélicos, los ámbitos y contenidos, los objetivos y decisiones, las fuentes y metodologías y las normativas. El artículo también señala el importante papel en la investigación sobre este tema que han tenido y tienen los profesores del área MIDE de las universidades españolas y la gran cantidad y calidad de las publicaciones sobre ello recogidas en las revistas científicas promovidas por su asociación investigadora AIDIPE.

Palabras clave: profesorado; evaluación; consecuencias; calidad.
\end{abstract}

Correspondencia: Tomás Escudero Escorza, tescuder@unizar.es, Universidad de Zaragoza, Departamento de Educación, Instituto de Ciencias de la Educación, C/Pedro Cerbuna, 12, 50009, Zaragoza (España). 


\begin{abstract}
Teacher evaluation is a very relevant area of research in education. It offers a great potential to strengthen features of educational quality whether it is carried out while being adequately contextualized, by following the principles and characteristics of the evaluation defended in numerous scientific publications of main international experts. In order to base the correct evaluation, important aspects such as the model approaches, the scopes and contents, the objectives and decisions, the sources and methodologies and the regulations are analysed. In addition, the important role of teachers in the area of Research Methods and Diagnosis in Education (MRDE) belonging to Spanish universities, as well as the large quantity and quality of publications on this subject in scientific journals promoted by his research association AIDIPE.

Keywords: teachers; evaluation; consequences; quality.
\end{abstract}

\title{
I. Introducción y contextualización de la evaluación del profesorado
}

En materia de desarrollo educativo y social en general, la investigación evaluativa ha ido tomando cada vez más relevancia en las últimas décadas como vehículo para asegurar y fortalecer la calidad (Escudero, 2003, 2006a, 2016a; Weiss, 1998) y también es cierto que el ámbito educativo y sus planteamientos teórico-prácticos han sido claves para el desarrollo y fortalecimiento de la investigación evaluativa (Escudero, 2005-2006, 2006b, 2010, 2011).

Esta realidad ha sido manifiesta entre el profesorado de nuestra área de Métodos de Investigación y Diagnóstico en Educación (MIDE), con muchísimos ejemplos de investigaciones y publicaciones relacionadas con la evaluación de programas e instituciones educativas, profesores y otros aspectos relevantes de la educación. Las dos importantes revistas (Aliaga, 2014) de nuestra Asociación Interuniversitaria de Investigación Pedagógica (AIDIPE), Revista Electrónica de Investigación y Evaluación Educativa (RELIEVE) y esta Revista de Investigación Educativa (RIE) son un magnífico ejemplo de dicha realidad y un buen instrumento para fortalecer el conocimiento y situación de la investigación evaluativa y la calidad en educación (Muñoz \& Espiñeira, 2010). En un reciente análisis bibliométrico de RIE entre 2000 y 2012, se demuestra que la evaluación de programas, centros y profesores es la temática más tratada, con el 18,6\% de los artículos (Ariza \& Quevedo-Blasco, 2013). Un ejemplo importante es el vol. 18, no 2 de RIE del año 2000, número monográfico sobre evaluación de programas educativos, coordinado por nuestro querido compañero el profesor Ramón Pérez Juste, en el que aparecen 35 artículos mayoritariamente de profesores del área MIDE.

La evaluación de personal es uno de los ámbitos fundamentales de la investigación evaluativa y en educación son los profesores el objeto más importante y con más tradición y esfuerzos de este ámbito investigador, por su relevante incidencia en su calidad y de los sistemas e instituciones educativas. Pero no debemos olvidar que es también una temática de las más conflictivas para su puesta en marcha y la toma de decisiones a posteriori. Algunos conocidos investigadores en este terreno declararon que existían pocos ejemplos prácticos de evaluación de los profesores que 
puedan considerarse correctos y efectivos (Scriven, 1981; Wise, Darling-Hammond, McLaughlin \& Bernstein, 1985) y de ahí la importancia que tiene que el profesorado tenga un pensamiento adecuado y una conducta positiva con su evaluación (Mateo, Escudero, De Miguel, Mora \& Rodríguez, 1995, 1996a), mostrando interés sobre los procedimientos que se utilizan y los que deberían utilizarse, para beneficiarse de su evaluación (Nevo, 1994), romper la imagen de la evaluación como algo contra el profesorado, sustituyéndola por otra a su favor y de su actuación profesional.

La investigación ha demostrado que la evaluación del profesorado es un camino directo hacia la mejora educativa, pero teniendo siempre en cuenta que se trata de un proceso tan importante como delicado (Escudero, 2016b, 2018; Mateo, Escudero, De Miguel, Mora \& Rodríguez, 1996b; Villa, 1985), pues es necesario establecer y respetar claramente los objetivos de la evaluación, seleccionar y aplicar correctamente desde el punto de vista académico y social las metodologías, las técnicas y los indicadores empleados en la misma y contextualizar adecuadamente el proceso y la valoración de los resultados. Los problemas se suelen centrar en la definición de los criterios de calidad del profesorado, en los propósitos y consecuencias de la evaluación y en la aceptación y colaboración del profesorado. El problema es más práctico que teórico, pues, aunque pueden existir todavía algunos problemas de fundamentación de la evaluación, el panorama es más rico y sólido que las prácticas evaluadoras (Mateo, 1993).

La actividad profesional de todos los individuos, y por supuesto la de los profesores, se puede evaluar desde dos perspectivas, desde sus destrezas y desde los resultados de su trabajo. Las primeras se suelen configurar como las características y cualidades personales, por una parte, y como las conductas que muestra y actividades que realiza. Además, se examinan los resultados de su actividad. Los sistemas de evaluación se focalizan en una perspectiva o en otra, o en alguna combinación de ambas (Escudero, 2005 , 2006a). En esta práctica surge el debate sobre el uso del rendimiento de los alumnos como medida principal de la efectividad del profesor, defendido por administradores y políticos, pero no por los expertos en evaluación, pues no es responsabilidad total del profesorado porque no tienen control directo de ello, pues depende en gran medida de las características y actividad de los alumnos. Por ello, estas puntuaciones deben utilizarse siempre delicadamente y con otras medidas de la evaluación.

Lo que sí pueden controlar en gran medida los profesores es el desarrollo de sus destrezas hasta donde permitan sus capacidades y sus conductas profesionales. Estas destrezas y conductas no aseguran totalmente la calidad del sistema educativo, pero sí son una condición necesaria. Además, su evaluación sí que ofrece pautas para introducir mejoras y cambios, algo que, como indican Stufflebeam y Shinkfield (1987), es el propósito más importante de la evaluación. Lógicamente, las cualidades y las conductas de los profesores son un contenido obligado en su evaluación, sobre todo si tiene alguna orientación formativa, pero también en los enfoques sumativos, sobre todo en los de tipo promocional. También es importante para las evaluaciones que las recompensas no sean limitadas en número, que se fortalezcan los incentivos para el trabajo en equipo, que se analice la adecuación de las competencias y conductas con el contexto en el que se actúa y que se evite limitar la validez del diagnóstico evaluativo, sustentándolo de manera excesiva o única en datos cuantitativos. 


\section{Modelos de evaluación}

La evaluación de los profesores se lleva a cabo con diferentes modelos de fundamentación teórica y aproximación práctica, según las circunstancias concretas de la evaluación que se trate (Marczely, 1992).

La identificación y valoración de los rasgos y conductas del profesorado es un modelo evaluativo importante y frecuente para describir la enseñanza efectiva y las conductas específicas relacionadas con la buena instrucción. Lógicamente, este enfoque cuenta con la posibilidad de apoyo de escalas de evaluación cuantitativas y cualitativas.

La evaluación centrada en los objetivos instructivos es otro modelo relevante y sugiere vías para la mejora (Popham, 1973), pero su utilización es discreta porque el rendimiento académico de los alumnos desborda el control completo del profesorado y, consecuentemente, no es bien aceptado por el profesorado como criterio evaluador.

La evaluación del proceso de enseñanza, se apoya en la consideración de los aspectos que la investigación ha venido mostrando como enseñanza efectiva, tales como preparación, diseño, objetivos, métodos, control de aprendizaje, práctica guiada, práctica independiente, etc. Este modelo no es muy utilizado, pero, como dice Wolfe (1987), tiene suficientes potencialidades como para tener una mayor utilización.

Boyan y Copeland (1978) describen el modelo centrado en las preocupaciones del profesor como una oportunidad para que los profesores analicen su propio progreso como profesionales y busquen apoyos para la mejora. Los profesores evalúan su propio rendimiento y establecen sus propios objetivos a partir de ello. La autoevaluación es un elemento clave en el modelo.

La mezcla de dos o más enfoques de evaluación conforma el denominado modelo ecléctico, importante y útil para tomar decisiones de mejora.

Scriven (1995) analiza los diferentes enfoques de evaluación del profesorado, reconociendo que todos tienen algunos problemas y resaltando que los que tienen más probabilidad de éxito son los modelos híbridos y pragmáticos, en los que se mezclan distintos modelos simples, tanto de método como de criterio. Este autor resalta la evaluación de los deberes genéricos del profesor y los específicos del tipo y del puesto de trabajo concreto en el que se está ejerciendo la docencia. En su guía teórico-práctica de evaluación de profesores, Shinkfield y Stufflebeam (1997), ofrecen también un amplio listado de modelos de evaluación de profesores elaborado en el CREATE (Center for Research on Educational Accountability and Teacher Evaluation), que son denominados por su característica más distintiva.

Con independencia de la aproximación modélica que se tome, conviene tener en cuenta algunas orientaciones básicas para fortalecer la calidad de la evaluación. Peterson (1995), por ejemplo, resalta como importantes el énfasis de la buena docencia, las razones de la evaluación, la integración del profesorado en la acción evaluadora, la utilización de diversos evaluadores, limitando las funciones de los directivos administrativos y la utilización de suficientes fuentes, variables y tiempo para fundamentar adecuadamente los juicios sobre los profesores. 


\section{3. Ámbitos de actuación y decisión de contenidos en la evaluación}

Buena parte de las complejidades que rodean la evaluación de profesores se deben a la multiplicidad de ámbitos en la que debe tener lugar y de decisiones que debe fundamentar. Stufflebeam (1991) señala que estos ámbitos están en el terreno de la preparación, práctica y perfeccionamiento profesional de los profesores y, dentro de cada uno de ellos, en aspectos relacionados con la selección, participación y resultados de los planes específicos en cada caso. Una síntesis de todos estos ámbitos es recogida en el capítulo de fundamentación teórica de nuestro estudio de la evaluación del profesorado de secundaria (Mateo, Escudero, De Miguel, Mora \& Rodríguez, 1996a), señalando el conjunto de los más importantes ámbitos de actuación y de decisión en la evaluación de profesores y, en general, de todo el personal educativo. Sin embargo, es evidente que no todos los ámbitos son igual de importantes y relevantes en cuanto a su significado en materia de planificación educativa. En general, cuando se habla de evaluación de profesores, se enfatiza especialmente la evaluación relativa al área de la práctica profesional, pero es importante aclarar que, en conjunto, la evaluación de profesores es mucho más que la evaluación de su práctica y que también incide en su formación previa y en su perfeccionamiento y formación continuada.

La identificación de los contenidos apropiados para la evaluación es una faceta crítica y una tarea especialmente compleja desde los puntos de vista conceptual y empírico (Dwyer, 1994). La clave de este problema está en la falta de un criterio único de definición de buen profesor, que permita focalizar perfectamente su evaluación. Consecuentemente con lo anterior y aun dentro de las imprecisiones apuntadas, la práctica de la evaluación se mueve alrededor de contenidos ubicados en tres ámbitos claros, lo que el profesor es, lo que hace y lo que consigue. Los tres ámbitos tienen sus limitaciones, pero los tres son considerados importantes y complementarios por la falta de ese criterio nítido de buen profesor. El ámbito de lo que el profesor es, de lo que sabe, es el ámbito de su competencia, el de lo que hace es el ámbito profesional y el de lo que consigue es el ámbito de la productividad, entendida esta como contribución al aprendizaje del alumno y no como rendimiento del alumno (Schalock, Cowart \& Staebler, 1993).

Partiendo de estos planteamientos se han ofrecido en la literatura muchos listados de aspectos que deben ser valorados en la evaluación de los profesores. Así, por ejemplo, Scriven (1988 y 1994) llevó a cabo un análisis detallado de los deberes del profesor que describen su trabajo, su profesión, y que deben ser el contenido básico en su evaluación. Los deberes señalados son: el conocimiento de la materia, la competencia instruccional en comunicación, gestión, programación y desarrollo, la competencia evaluativa, la actitud profesional y utilización de los recursos disponibles y la relación con el centro y la comunidad.

Una de las aproximaciones más completas en este terreno es la ofrecida por Schalock et al. (1993), partiendo del análisis de productividad y efectividad docente y de la necesaria revisión de estos conceptos, enmarcando los contenidos que se tienen en cuenta en la evaluación de los profesores. Estos conceptos son los siguientes: 
A. Conocimiento docente. Preparación en aquellas materias relacionadas con su profesión. Materia a enseñar, pedagogía, psicología del desarrollo, etc. Se evalúa con tests y entrevistas.

B. Destrezas docentes. Medios que los profesores usan para aplicar su preparación para la docencia; métodos y técnicas didácticas, estímulos, etc. Se evalúa observando la conducta en el aula o en situaciones de simulación.

C. Competencia docente. Preparación específica para cumplir de manera adecuada con las responsabilidades y las funciones profesionales tales como diseño y planificación docente, motivación de los estudiantes, orientación y control del progreso de los estudiantes, trabajo con los colegas, administradores y padres. Se evalúa observando a los profesores y a sus alumnos en el contexto escolar y con entrevistas a colegas, directivos, padres y estudiantes.

D. Efectividad y Productividad docente. La contribución del profesor a las ganancias de los estudiantes dentro de un área concreta de contenidos y en todo el ámbito formativo, en un cierto periodo de tiempo. Se evalúa con distintas medidas del aprendizaje y de la formación de los alumnos, analizando las ganancias, debidamente contextualizadas e interpretadas.

E. Profesionalidad docente. Seguimiento de una conducta propia de un verdadero profesional de la docencia: cumplimiento profesional, trabajo con colegas, directivos, padres, servicios a la comunidad escolar, integración en actividades de perfeccionamiento profesional, autocrítica profesional, etc. Se evalúa a partir de documentos, entrevistas e informes de distintas fuentes.

Estos ejemplos de potenciales contenidos para la evaluación de profesores no agotan los análisis que ofrece la literatura especializada, pero la gran mayoría de los planes de evaluación que pueden analizarse estudian aspectos recogidos en estos listados.

\section{Propósitos y consecuencias de la evaluación del profesorado}

Siempre ha sido reconocida por los expertos la multiplicidad de propósitos y razones por las que se pone en marcha la evaluación del profesorado y las diversas consecuencias que puede tener (Genova ,1976; Nevo, 1994).

Una síntesis de los diversos propósitos recogidos en la literatura con los que se lleva a cabo la evaluación del profesorado, nos lleva a citar, al menos, los siguientes: 1) El desarrollo profesional de los docentes, mejorando su competencia, su acción didáctica y su rendimiento docente, convirtiéndose en definitiva en mejores profesores, 2) El control de su cumplimiento profesional, 3) El reconocimiento social de su labor, informando a la sociedad del rendimiento del profesorado y de los centros 4) La incentivación personal (económica, horarios, permisos), 5) Las ayudas a determinados proyectos y actividades, 6) El reconocimiento y la promoción profesional (carrera docente), 7) La selección de cargos y responsables docentes, 8) La mejora de la calidad de la enseñanza (mejora del centro y del sistema educativo), 9) La exigencia de responsabilidades (rendición de cuentas), 10) La autocrítica del profesorado, 11) El establecimiento de sanciones, 12) La selección de profesores, 13) Elaborar y mantener un inventario de recursos personales para reajustes y perfeccionamiento, 14) Mejorar 
el currículum, el programa y la acción departamental, 15) Mejorar la orientación al alumnado en la selección de especialidades y disciplinas,. Todos estos propósitos no son igualmente relevantes, pero es habitual que en los programas de evaluación exista más de un único propósito y más de una única consecuencia.

Denham (1987) agrupa estos múltiples propósitos en dos grandes bloques, selección y asistencia. El primer propósito, la selección, está en la raíz de todo sistema de incentivos al profesorado, mientras que el segundo propósito, la asistencia, se relaciona con la ayuda directa a los profesores en su trabajo y en su desarrollo profesional, a veces de una manera formal, con un entrenamiento organizado y otras de manera más informal, a través de consejos y asesoramiento de personas experimentadas. Dentro de este planteamiento, Natriello (1990) nos habla de tres propósitos en la evaluación del profesorado: 1) Influir en el rendimiento en su actual puesto profesional persiguiendo la mejora, 2) Controlar y conducir el movimiento de los profesores entre distintos puestos profesionales y 3) Legitimar el procedimiento de control del sistema, proporcionando un sentimiento de justicia y equidad en la gestión de los derechos y deberes de todos los implicados. En línea similar con la anterior, aunque con ciertos matices diferenciales, De Miguel (1998) señala tres enfoques de utilidad para la evaluación de los profesores, como rendición de cuentas, como estrategia de promoción, y como herramienta para la mejora.

Un buen sistema de evaluación de los profesores en un centro es un importante elemento de legitimación y de credibilidad para la organización y sus miembros ante su entorno, además de un ejemplo de responsabilidad. Lo mismo podemos decir si pensamos en sentido contrario, esto es, en el hecho de que resulta inadecuado hablar de la evaluación individual de un profesor, al margen de la institución y el contexto educativo en el que actúa. Por ello, la evaluación del profesorado debe estar integrada en la evaluación institucional (De Miguel, 1998), planteándose con perspectiva colegial, en el marco de unos propósitos, unos recursos y unos procesos compartidos.

\section{I. Selección y estabilidad del profesorado}

Hablamos, sin duda, de uno de los objetivos claves para la evaluación. Es crítico seleccionar bien a los profesores en cuanto a competencia y a disposición docente, aunque sabemos que se trata de una tarea compleja y delicada. En muchos momentos y en no pocos lugares, el acceso a la función docente no ha necesitado otro requisito previo que la posesión de un título universitario (Haney, 1990), pero, este proceso se ve periódicamente convulsionado por desajustes acusados entre oferta y demanda de plazas.

Centrándonos en la selección adecuada de profesores, esto es, en el proceso de elección del mejor candidato entre varios, o de comprobación de que un candidato es suficientemente bueno para seleccionarlo, o de ambas cosas a la vez, nos encontramos cuestiones claves para el proceso de evaluación que nos conduzcan a la selección acertada (Scriven, 1990). Una gran pregunta es ¿qué debemos evaluar? y la respuesta es su valía (su mérito en relación con el tipo de trabajo, más su valor específico para la institución para la que se selecciona). Un profesor que enseña muy bien tiene mucho mérito, un profesor que enseña algo que nadie puede o quiere enseñar, pero que obliga- 
toriamente tiene que ofrecerse, tiene mucho valor (Scriven, 1995). Sin embargo, mérito y valor no son siempre utilizados de manera adecuada. Los méritos profesionales, que se evidencian con lo que se ha hecho en el pasado, no siempre son presagio de productividad en el futuro, aunque sean un importante indicador de la competencia personal. El valor de un candidato se aprecia a la vista de las misiones y necesidades de la institución para la que se selecciona. Al contrario que mérito, que es un indicador absoluto, valor es un indicador relativo, a menudo imposible de explicitar en las convocatorias de selección; sobre todo si son generales. El mérito siempre tiene las dimensiones de calidad y cantidad, el valor se mueve en ámbitos de valoración más sutiles y contextualizados. La clave de la selección está en la búsqueda del adecuado balance entre mérito y valor, algo no siempre fácil. Una postura eficaz es la de asegurar que la selección se produce solamente entre candidatos con suficiente mérito, introduciendo el concepto de valor en la segunda y definitiva fase de la evaluación, a la búsqueda del candidato más valioso para la institución. El valor es un elemento fundamental de consideración cuando se trata de crear un nuevo puesto de profesor y en la continuidad de plazas.

Naturalmente, la valía de los profesores no se evalúa en abstracto, sino que se deben analizar determinadas características y competencias de los profesores ya señaladas (conocimientos académicos, pedagógicos, destrezas docentes, entusiasmo docente, etc.), utilizando los instrumentos y procedimientos adecuados en cada caso. La decisión, selección o no, requerirá seguramente un juicio cualitativo, aunque sea a partir de, en gran medida, indicadores cuantitativos. El establecimiento de la valía, a la manera descrita, difícilmente puede ser otra cosa que un juicio de valor fundamentado con todo tipo de datos.

\subsection{La mejora de los profesores}

La evaluación de los profesores con orientación formativa, buscando el camino para que mejoren como profesionales es una de las principales preocupaciones de los expertos en evaluación y de los responsables educativos. El que los profesores mejoren sistemáticamente su competencia termina siendo la operación más eficiente para el sistema, pero no es la orientación más sencilla y barata de la evaluación, pues se necesitan recursos, evaluadores expertos y creíbles y una organización que posibilite la mejora. Además, es una evaluación absolutamente inviable sin la participación de los evaluados.

Esta evaluación se condiciona por el tipo de profesores implicados y de su experiencia previa. Peterson (1990), por ejemplo, señala que los profesores noveles, no suelen ser tratados de manera diferenciada, salvo para ser encargados de lo que no quieren los demás y, sin embargo, suelen tener unas necesidades específicas que requieren más ayudas con un énfasis diferente que las que se deben proporcionar a los compañeros más experimentados. Las principales necesidades de los profesores noveles se centran fundamentalmente en las áreas de la planificación y organización de la enseñanza, en la motivación y en el tratamiento de las diferencias individuales y en las prácticas evaluadoras (Veenman, 1984). 
La evaluación dirigida al crecimiento profesional de los docentes, en busca de la excelencia, es otro gran bloque de la evaluación formativa de los profesores, con un conjunto muy amplio de potenciales áreas y ámbitos de incidencia. Según Riegle (1987) este progreso profesional puede tener lugar en cinco grandes áreas: a) el desarrollo instruccional que enfatiza las destrezas técnico-pedagógicas, b) el desarrollo profesional que enfatiza la mejora personal dentro de su tarea profesional, c) el desarrollo de la organización que enfatiza las necesidades y prioridades institucionales, d) el desarrollo de la carrera que enfatiza la preparación para el ascenso profesional y e) el desarrollo personal que enfatiza la mejora individual en diversas facetas académicas, vitales, relaciones personales, etc. Naturalmente, este desarrollo profesional es inviable sin una implicación personal en el proceso evaluador del propio profesor, con expectativas de mejora y disposición para la innovación, experimentación y cambio docente.

\subsection{Incentivos y carrera docente}

Las mejoras salariales y profesionales constituyen otro gran bloque de los propósitos con los que se plantea frecuentemente la evaluación de los profesores, pero también aquí nos encontramos con algo problemático. No podemos olvidar que todo sistema de incentivos individuales o grupales será un instrumento débil y escasamente útil si no se crea en paralelo un sistema de oportunidades (Malen, 1999). El fundamento es que quien quiera mejorar tendrá la oportunidad de hacerlo y que, si lo consigue, será premiado por ello.

En este bloque nos encontramos con una idea básica que es la de premiar de manera diferenciada a los mejores (o los buenos) profesores, pero la compensación puede tener formas diversas. Necesitamos diferentes tipos de sistemas de incentivos, porque la motivación es un fenómeno complejo y las reacciones o respuestas a un determinado premio o sanción variarán de manera considerable (Malen, 1999). Básicamente hablamos de tres tipos de premios, esto es, compensaciones monetarias que pueden ser estables, afectando al salario o esporádicas, recompensas de tipo académico, licencia por estudios, ayudas para proyectos, permisos especiales, etc., y promociones de tipo profesional (Bacharach, Conley \& Shedd, 1990; Harry \& Greiner, 1985).

Las administraciones educativas ponen regularmente en marcha programas evaluativos que desembocan en incentivos en forma de compensaciones monetarias con el objetivo de optimizar el sistema educativo, mejorando la enseñanza, el rendimiento de los profesores y evitando la deserción de los mejores profesores, al relacionar sus retribuciones de forma más justa a sus rendimientos docentes (Hatry \& Greiner, 1985). Pero, por unas u otras razones, estos planes de «pago por mérito» suelen tener una vida corta (Bacharach et al., 1990).

El «pago por mérito» de los profesores es criticado con mucha frecuencia por utilizar procedimientos de evaluación con problemas de objetividad. Sin embargo, la búsqueda de mayor objetividad, apoyándose en indicadores de calidad más fielmente medibles, entra en cierta contradicción con un concepto global de competencia y rendimiento docente, en gran medida definido por cualidades y conductas que no se ajustan con ese tipo de indicadores. Además, el «pago por mérito» puede terminar siendo un elemento contra el trabajo cooperativo de los profesores, porque se compite por un incentivo 
monetario de un fondo habitualmente limitado. Sólo existen incentivos positivos para algunos y el resto se queda igual, pero los no incentivados se sienten penalizados. Las cuotas de ganadores producen efectos negativos en los «meritorios» que no son seleccionados para recibir el incentivo; desencanto, pasotismo y agresividades contra los seleccionados (Hatry \& Greiner, 1985).

Estos mismos problemas se producen en los sistemas de escalonamiento profesional, promoción de categoría dentro del mismo trabajo, porque siempre está sujeto a cuotas, que condicionan el ascenso por mérito o competencia (Bacharach, Conley \& Shedd 1986). La evaluación para carrera docente en sentido más amplio, donde a menudo el ascenso supone cambio de trabajo, profesor, director, inspector, etc., lleva aparejada algunos elementos de discusión importantes. ¿Qué competencia se debe evaluar? ¿La competencia docente, del trabajo del que se parte, o la competencia para el trabajo al que se aspira? El mérito de ser el mejor profesor no predice que se vaya a ser un director competente. Pero si elegimos a los que pueden ser potencialmente mejores directivos, probablemente no estemos premiando la competencia docente. Otro tema importante es que los sistemas que buscan la excelencia nunca pueden partir del principio de que ese terreno está limitado a unos pocos.

El ámbito de la promoción profesional del profesorado en el sistema universitario español, por ejemplo, es analizado de manera relevante e interesante en un reciente editorial de esta Revista (Jornet, 2017), señalando problemas en la fundamentación y los procesos que, según nuestra opinión, requieren obligatoriamente ciertas reformas para la mejora del sistema (Escudero, 2018). También en nuestras universidades, el pago por mérito e incentivo individual al profesorado es uno de los mecanismos empleados para intentar gestionar la calidad y su mejora de manera justa y eficaz. En un artículo ofrecemos un análisis de las potencialidades y contraindicaciones del procedimiento, así como la síntesis de una revisión metaevaluativa de la evaluación para incentivos individuales al profesorado, auspiciada por los gobiernos autonómicos, que desde hace unos años se ha generalizado en las universidades españolas (Escudero, Pino \& Rodríguez, 2010).

\section{Fuentes, métodos y estrategias}

Tras ver que los propósitos y los contenidos en la evaluación son múltiples y diversos, es lógico señalar que el proceso de evaluación podrá y deberá contener una amplia variedad de elementos metodológicos diferentes. Las fuentes, los procedimientos, los agentes y las estrategias evaluativas deben ser acordes con los contenidos y con los propósitos, teniendo presente siempre que muchos de los instrumentos empleados en la evaluación se han diseñado con un fin específico y no con varios a la vez (Denham, 1987). La evaluación de los estudiantes, por ejemplo, es cuestionada por muchos autores cuando se pretende promocionar o despedir profesores (Wilson \& Wood, 1996).

Los expertos aceptan que todas las aproximaciones metodológicas son útiles en algún momento y para alguna faceta evaluativa y que todas tienen sus limitaciones y que en la práctica se requieren generalmente aproximaciones metodológicas diversas y complementarias. En gran medida, una de las tareas más importantes del evaluador es sugerir la selección en cada caso de las fuentes, los procedimientos y los métodos que comentamos. 
Mehrens (1990) defiende que para evaluaciones que tienen propósitos formativos, de desarrollo profesional, por ejemplo, puede que no sea necesario combinar datos diversos e incluso que puede que sea mejor tratarlos separadamente. Sin embargo, cuando se trata de tomar decisiones de tipo sumativo, selección, acreditación, estabilidad, incentivos salariales, etc., parece obligado tomarlas a partir de una combinación de diversos datos e informaciones, según qué evaluación se trate. La combinación de datos puede hacerse con una perspectiva estadística o se puede hacer una apreciación global de todo el conjunto de las informaciones, como defiende Popham (1987). Cuando se trata de combinar datos y no de apreciaciones globales, se puede seguir el modelo de conjunción de datos, según el que los evaluados deben superar cierto nivel en todas las variables. La posición contraria sigue con el modelo de distinción, cuando existen varias medidas de una misma variable y se requiere la superación de alguna de ellas. En los procesos de selección y de empleo de profesorado se suele utilizar un modelo de compensación, según el que el fallo en alguna de las variables o criterios requeridos puede ser compensado con un buen rendimiento en los otros.

Como fuentes generalmente recomendables para la evaluación de profesores destacan según Peterson (1995), los informes de los estudiantes, de los directivos y de las familias de los estudiantes, las revisiones de colegas y expertos, el rendimiento de los estudiantes, ciertas pruebas a los profesores, la observación sistemática en el aula y la documentación (carpetas y dossiers de los profesores).

\section{I.Tests y pruebas a los profesores}

Examinar a los profesores de sus destrezas básicas, conocimientos pedagógicos y académicos, etc., a través de tests y pruebas de rendimiento, es un procedimiento de cierta utilización en su evaluación. Como señala Peterson (1995), es un procedimiento que debe considerarse como una fuente opcional para documentar la calidad del profesor. Los tests convencionales de elección múltiple son un procedimiento eficiente para la medición de ciertos niveles del conocimiento y competencia de un docente, pero existen destrezas docentes importantes que difícilmente pueden ser valoradas a través de un test de elección múltiple, por lo que se necesitan también otras pruebas abiertas de rendimiento (Mehrens, 1990).

\subsection{Observación en el aula}

Stodolsky (1990) critica omitir la observación directa en la evaluación de profesores y se pregunta ¿Cómo se puede evaluar a los profesores sin verlos en acción? Pero no podemos defender que observando a los profesores en sus aulas tenemos suficiente, pues se escapan muchas facetas importantes en su evaluación. Este procedimiento es a veces difícil de aplicar, pero la aparición del video y de los circuitos cerrados de TV ha ampliado considerablemente las posibilidades de la observación en el aula, que tal como defiende Murphy (1987) puede y debe ser formal, preparada de antemano, o informal, sin planificación previa, con participación de observadores diversos recogiendo información sobre muchos aspectos de la práctica docente. La identidad y la preparación de los observadores es otro elemento importante en la utilización de este 
procedimiento de evaluación. La preparación técnica deberá ser mayor cuanto más elevado sea el nivel de inferencias necesario para rellenar los protocolos de observación. Los observadores más habituales suelen ser inspectores, supervisores y directivos, pero también se utilizan otros colegas. En general se recomienda la mezcla de supervisores y colegas (Hatry \& Greiner, 1985).

\subsection{Escalas e informes de evaluación}

La utilización de escalas de evaluación cumplimentadas por diversos agentes, tiene larga tradición en el ámbito de la evaluación de profesores. Las primeras escalas se diseñaron para utilización de los administradores, supervisores y directivos, pero después se amplió el rango de agentes con los estudiantes, inspectores e incluso los propios profesores. Estas escalas utilizan tanto técnicas cuantitativas como cualitativas. A veces se valoran rasgos, otras se ordenan y en algunos casos se limitan a señalar la presencia o no de una característica o circunstancia. Los contenidos o dimensiones de análisis de estas escalas suelen ser generalmente: a) dirección de la clase, b) destrezas instructivas, c) personalidad docente, d) preparación académica y profesional, e) esfuerzo para la mejora, f) interés en el trabajo y g) habilidad para la colaboración con otros (Barr \& Emans, 1930).

En paralelo a estas escalas para utilización de responsables educativos, últimamente ha crecido la utilización de escalas o cuestionarios de evaluación de los profesores por los clientes del proceso educativo, esto es, estudiantes, ex-alumnos y, en menor grado, padres. Sobre estas escalas, su utilización, en la educación superior, sobre todo, sus ventajas, problemas, etc., existe abundante literatura especializada (Abrami, 1989; Marsh \& Hocevar, 1991) que evidencia que estos procedimientos son muy valiosos en la evaluación de los profesores, sobre todo en procesos con orientación formativa. La valoración de los estudiantes es la principal fuente de información cuando analizamos el desarrollo y la motivación en la clase, las oportunidades para aprender, el grado de afecto y comunicación desarrollado entre profesor y estudiante y la equidad en el aula. Además, el estudiante proporciona una información directa sobre los textos, los exámenes y los trabajos del curso. Se trata de una fuente eficiente sobre muchos aspectos del profesor y su docencia en general pero tiene algunas limitaciones, pues los estudiantes no son expertos en la materia enseñada, ni tampoco en aspectos pedagógicos, lo que puede perjudicar su perspectiva global. Como complemento a los cuestionarios a los estudiantes, que es la manera más frecuente de recoger su opinión, se usan técnicas como entrevistas grupales o los grupos de discusión. En conjunto, es incuestionable el valor de la opinión de los estudiantes para evaluar a sus profesores, fundamentalmente para la orientación formativa y menos para la sumativa, pues el proceso es importante, pero tiene algún riesgo (Escudero, 1999, 2000).

\subsection{Rendimientos de los alumnos}

No hay ninguna duda de que los resultados de los alumnos sí son una fuente importante dentro de la evaluación del profesorado y de la docencia, aunque resulta un elemento discutible y discutido como criterio de competencia y de efectividad 
docente, pero es obligado utilizar el rendimiento de los alumnos, pues proporciona una información valiosa para la toma de decisiones (Stiggins \& Bridgeford, 1985) y lo lógico es apoyarse fundamentalmente en las ganancias de los alumnos a lo largo de un periodo de tiempo y, finalmente, contextualizar debidamente el significado de las puntuaciones para posibilitar su análisis comparativo (Murphy, 1987). El soporte de este criterio está en la idea de que el objetivo final del proceso evaluativo es asegurar la calidad del aprendizaje de los alumnos y esto sólo se puede comprobar examinando sus rendimientos académicos combinados con otros criterios y refiriéndolos al nivel escolar en conjunto más que al nivel de cada profesor individual (Glass, 1990). La utilización del rendimiento de sus estudiantes para evaluar a un profesor es algo que parece lógico para mucha gente, especialmente para los no educadores. Quizás por ello, un sistema de evaluación del profesorado que no incluya el rendimiento de los estudiantes puede resultar poco creíble para muchas audiencias, aunque sepamos que la calidad y el esfuerzo del profesor no siempre tienen una conexión directa con el aprendizaje del estudiante, pues existen muchos condicionantes intermedios.

\subsection{Autoevaluación de los profesores}

La autoevaluación tampoco es un procedimiento habitual en muchos programas de evaluación de los profesores (Stiggins \& Bridgeford, 1985), sin embargo, es claro que se trata de una importante técnica para la evaluación formativa (Barber, 1990). La autoevaluación se trata de reflexiones del profesorado sobre sus creencias educativas, sus prácticas y sus logros escolares (Airasian \& Gullickson, 1994), algo fundamental para mejorar profesionalmente, porque conlleva una decisión consciente de autovaloración crítica, algo difícilmente explicable si no se desea perfeccionarse y corregir las deficiencias encontradas. La autoevaluación se apoya en diversas técnicas. Carroll (1981), por ejemplo, habla de cinco procedimientos básicos: las escalas de autovaloración, los autoinformes, el estudio de los propios materiales, la observación de la docencia de colegas y el análisis a través del video de la propia práctica docente. La autoevaluación tiene ciertas limitaciones técnicas que se superan si se utiliza exclusivamente para decisiones formativas y se complementa con otras fuentes y procedimientos.

Personalmente, por mi actividad en comisiones de evaluación de profesores en la Agencia Nacional de Evaluación de la Calidad y Acreditación (ANECA), he de defender el gran valor que tienen los autoinformes del profesorado para valorar razonablemente y de manera acertada sus méritos y actividades. Lo mismo podemos decir de la evaluación institucional que se fortalece, se racionaliza y se facilita claramente compaginando la autoevaluación (la evaluación interna) con la evaluación externa de expertos y afectados.

\subsection{El portafolio y el dosier del profesor}

Últimamente ha aparecido con fuerza la utilización del portafolio del profesor en el terreno de su evaluación (Seldin, 1997), aunque con cierta controversia. El portafolio es un archivo personal del profesor donde guarda sus materiales, documentos y apoyos didácticos de todo tipo. Su utilización como instrumento de evaluación obliga 
al profesor a tener un especial cuidado del citado archivo. La profesora Klenowski (2007) defiende que el uso del portafolio como método evaluativo es bueno para la formación y mejora del profesorado y de sus actuaciones y métodos didácticos, pero lógicamente, se requiere en el mismo unos contenidos adecuados. Seldin (1997) analiza este tema y recomienda recoger más de una decena de aspectos concretos relativos a la docencia y relacionados con ella.

También hay evaluadores que defienden que el dosier es un procedimiento de evaluación sumativa de los profesores que tiene ventajas sobre el portafolio (Peterson, Stevens \& Mack, 2001), pues dicen que es más fácil de juzgar, de fiabilidad demostrable y menos costoso. El dosier es una colección de documentos relacionados con una materia específica, incluyendo muchas de las ventajas del portafolio, pero con longitud limitada. El dosier es más compacto pues no es una simple colección de documentos e información, pues esta debe ser sintetizada y revisada antes de entregar a los evaluadores. En tal sentido, se trata de un procedimiento más útil para las decisiones sumativas, mientras que el potencial del portafolio es muy útil para las decisiones formativas.

\subsection{La revisión de colegas o pares}

Es un proceso en el que los profesores, utilizando su propio conocimiento y experiencia, examinan y juzgan el mérito y el valor de la práctica docente de compañeros de otros contextos, lugares o centros. Su ventaja es que puede proporcionar una buena información sobre el conocimiento real que se tiene de la disciplina y cómo se organiza curricular y didácticamente, pero puede tener problemas de fiabilidad, pues es un procedimiento con escaso bagaje de investigación detrás y la poca disposición que tiene el propio profesorado a participar en procesos de este tipo. La revisión de materiales de instrucción es la tarea más fiable de todas, pero limitarse a ella tiene el problema de que puede producir una visión inexacta del rendimiento docente del profesor pues la actuación docente puede ser muy diferente a partir de materiales similares. Peterson (1995) nos ofrece algunas sugerencias sobre los aspectos que deberían ser analizados en este tipo de evaluación.

\subsection{Informes de padres y familiares}

Los padres y familiares también pueden ser una audiencia significativa para valorar el rendimiento de los profesores, a través de sondeos, entrevistas o grupos de discusión (Epstein, 1985), pues, aunque no tienen una visión directa de lo que ocurre en el aula, sí que la tienen de la conducta con ellos mismos y de las reacciones de los estudiantes a su conducta, cuando no están en las aulas.

\section{Normas en la evaluación de profesores}

En 1975 se estableció en Estados Unidos un comité profesional de normas para la evaluación educativa en el que participan asociaciones de profesores, de administradores, de consejos escolares y de expertos en evaluación y que unos años después publicó unas normas para la evaluación de programas, proyectos y materiales (Joint 
Committee, 1981) y después elaboró unas normas para la evaluación de personal educativo (Joint Committee, 1988), manteniendo los cuatro atributos básicos adoptados para la evaluación de programas, esto es, legitimidad, utilidad, factibilidad y precisión, analizadas con todos sus detalles en el texto.

Las normas de legitimidad buscan la protección de los derechos de las personas afectadas por la evaluación, asegurando que esta se lleva a cabo ética y legalmente, en beneficio de los evaluados y los que se ven afectados por sus consecuencias. Las normas de utilidad se dirigen a buscar que las evaluaciones de los profesores tengan influencia y consecuencias, para la mejora de su actividad profesional y para que presten un excelente servicio a los educandos. Las normas de factibilidad hablan de una evaluación eficiente y realista, fácil de utilizar, fundamentada adecuadamente y políticamente viable. Las normas de precisión requieren que la información sea técnicamente fiable y válida y una correcta descripción del trabajo evaluado.

La aplicación razonable y eficiente de estas normas para la evaluación de profesores fue un tema de interés, trabajo y producción científica para un gran experto en evaluación como Stufflebeam y algunos de sus colaboradores (Stufflebeam \& Brethower, 1987; Stufflebeam \& Sanders, 1990).

\section{Práctica evaluativa para la mejora. Problemas y aspectos relevantes}

La evaluación del profesorado, como factor esencial de mejora del sistema educativo, adquiere su verdadera relevancia cuando tiene una plasmación práctica adecuada. El mayor campo de operaciones y de prácticas iniciales en este terreno ha sido el sistema educativo americano, pero la práctica se ha internacionalizado y extendido a una mayoría de sistemas educativos como por ejemplo los europeos, que también tienen formatos interesantes (Mateo et al., 1996a). Sin embargo, no existen soluciones satisfactorias en todos los países, pues en algunos casos su aplicación ha sido problemática (McLaughlin, 1990). Tres son los tipos de problemas que pueden incidir en el bloqueo práctico: Problemas técnicos en las estrategias y recursos de la evaluación, que producen dudas a los diferentes usuarios de la evaluación, Problemas de gestión política de la evaluación, por su inserción poco adecuada en el sistema educativo y Problemas de legitimación de la evaluación, por falta de una cultura adecuada entre el profesorado sobre el tema, pues como señala Harris (1986) el reto de un programa de evaluación no se ubica en el terreno meramente técnico. Un modelo de evaluación del profesorado será tanto más efectivo cuanto mayor sea el acuerdo que tenga detrás, pues la confianza entre profesores y responsables educativos es condición sine qua non para que un sistema de evaluación funcione. En la práctica evaluativa hay que dejar muy claro que el principal propósito es la mejora y el enriquecimiento profesional, rompiendo la imagen de que no se trata de algo para perjudicar al profesorado. Frase y Streshly (1994) sugieren dos grandes recomendaciones para asegurar la calidad evaluativa: que la política evaluadora debe ser clara y conocida por todos los implicados y que la mejora del sistema es posible sólo si existe colaboración de todos los implicados. Otro importante principio es que se debe dejar tiempo al profesorado para que mejore, proporcionándole recursos, técnicas y ayudas, para que este objetivo funcione y se pueda potenciar el progreso profesional. 
Peterson (1995), al tratar los problemas en la evaluación de los profesores identifica tres áreas de reflexión, la técnica, la sociológica y la política, señalando que cualquier modelo o sistema de evaluación debe solucionar los problemas o las complicaciones que se presentan en cada una de estas áreas. En el área técnica hay que tratar adecuadamente las múltiples audiencias, las orientaciones formativa y sumativa, la naturaleza de los datos, las múltiples fuentes de información, los datos obligatorios y específicos, la evaluación del contexto y de otros elementos condicionantes o moduladores, el tiempo y la validez y fiabilidad. En el terreno sociológico hay que cuidar las relaciones con los responsables académicos y administrativos, los estudiantes, sus familias, los compañeros profesionales, etc. En tercer lugar, hay que destacar el carácter político de la evaluación y los problemas que en este terreno conlleva, que dependerán de la organización política general y del sistema educativo. En todo caso, hay que considerar adecuadamente los sistemas de poder y de toma de decisiones en los distritos y en las organizaciones educativas, la implicación de los propios profesores en el sistema de evaluación, las políticas de personal, de retención, de estabilización y de promoción y todo lo relativo a la política informativa y el tratamiento de la información generada con la evaluación.

\section{Revisión del rendimiento académico por los alumnos como criterio de evaluación de los profesores}

Ningún especialista pondría en duda el valor potencial del rendimiento académico de los alumnos, de los resultados del proceso de enseñanza-aprendizaje, como criterio de evaluación de sus profesores, pues se trata de un índice directo de calidad de la acción de los profesores, si se consideran los cambios producidos en los estudiantes durante el proceso y no solamente los resultados al final del mismo. Sin embargo, aun teniendo en cuenta el efecto concomitante de los antecedentes y de la situación inicial de los alumnos en los resultados, siempre existe la duda de si el rendimiento escolar es un criterio de evaluación justo, puesto que los profesores no son responsables de dicho rendimiento en su totalidad. Los profesores son responsables de su propia actuación, sin duda influyente en el rendimiento de los alumnos, pero no lo son de otros factores también influyentes, como por ejemplo el propio esfuerzo personal de los estudiantes. Lógicamente, no se le pueden pedir responsabilidades a un profesor de algo que no está bajo su control. Incidiendo en este problema, Peterson (1995) analizó críticamente la utilización del rendimiento de los estudiantes en la evaluación del profesorado, resaltando las ventajas e inconvenientes y las estrategias metodológicas más habituales. El estudio de relaciones entre características y conductas didácticas de los profesores y los resultados de sus alumnos es un tema relevante de investigación educativa (Gellmann \& Berkowitz, 1992), incidiendo en las características de calidad de los profesores. Resultados de este tipo de investigaciones en nuestra revisión (Escudero, 1980), vienen a indicar la falta de capacidad predictiva de variables tradicionales como méritos, expediente, etc., pero también indican la necesidad de unos niveles mínimos de preparación y conocimiento para poder ser un profesor competente. Un tema muy investigado es el de la estabilidad temporal de los profesores en su capacidad para promover el aprendizaje. Las revisiones de Rosenshine (1970) resaltaban que hay que 
ser cauteloso con la estabilidad temporal de la efectividad de los profesores, por lo que, si la estabilidad no está asegurada, parece obligado evaluar la efectividad de los profesores con cierta periodicidad (Escudero, 1980).

Incuestionablemente, el panorama que nos ofrece la investigación en este campo obliga a la prudencia en la utilización del rendimiento de los alumnos como criterio de evaluación de los profesores, aunque es un criterio relevante, que debe utilizarse con cautela y junto a otros criterios.

\section{Opinión de los profesores sobre su evaluación}

Cualquier programa de evaluación del profesorado debe tener un nivel razonable de aceptación para que se pueda llevar a cabo, pues no existe duda de la importancia que tiene la visión de los propios profesores sobre su propia evaluación para planificarla y desarrollarla.

Del análisis en nuestro país se desprende que nuestro profesorado no ve con malos ojos la evaluación de su trabajo, si se realza su orientación formativa o para procesos de selección. En cuanto a contenidos y agentes de la evaluación, hay una cierta diversidad entre los resultados en los diferentes estudios (Fernández, Navarro, Higuera \& Martínez, 1993; González, 1992; Mateo et al., 1995; Palacián \& Blanco, 1993).

\section{Reflexión final}

Este artículo presenta y defiende la evaluación del profesorado como un camino directo hacia decisiones para la mejora de la calidad educativa, pero, lógicamente, esto se hace señalando y analizando que es obligado el diseño preciso de los recorridos, la utilización de vehículos adecuados y un desarrollo apropiado del proceso que conduce a la toma de decisiones. Estos criterios y fundamentos metodológicos para hacer bien el recorrido de la evaluación son aspectos considerados y analizados en el artículo y en las múltiples publicaciones sobre la evaluación a las que se hace referencia en el mismo. Reforzando esta idea de calidad evaluativa, podemos citar también otros trabajos importantes de compañeros de nuestra área académica, que pueden también ser consultados como el conjunto de referencias para fortalecer la calidad de la evaluación (De La Orden, 1990; De Miguel, 1991; Escudero, 1989; García Ramos, 1997; Muñoz Cantero, Ríos De Deus \& Abalde, 2002; Pérez Juste, Cristobal \& Martínez, 1995; Rodríguez Espinar, 1997, 2003; Tejedor, 2003; Tejedor \& Jornet, 2008).

\section{Referencias}

Abrami, P. C., (1989). How should we use student ratings to evaluate teaching. Research in Higher Education, 30(2), 221-227. Recuperado de https://link.springer.com/article/10.1007/BF00992718

Airasian, P. W., \& Gulickson, A., (1994). Examination of teacher self-assessment. Journal of Personnel Evaluation in Education, 8(2), 195-203. Recuperado de https://eric. ed.gov/?id=EJ492227 
Aliaga, F. (2014). Editorial. Calidad y evaluación de las revistas científicas de educación. Revista de Investigación Educativa, 32(1), 11-12. Recuperado de http://www.redalyc. org/articulo.oa?id=283330505001

Ariza, T., \& Quevedo-Blasco, R. (2013). Análisis bibliométrico de la Revista de Investigación Educativa (2000-2012). Revista de Investigación Educativa, 31(1), 31-52. doi: http://dx.doi.org/10.6018/rie.31.1.160321

Bacharach, S. B., Conley, S., \& Shedd, J. B. (1986). Beyond career ladders: structuring teacher career development systems. Teacher College Record, 87(4), 563-574.

Bacharach, S. B., Conley, S., \& Shedd, J. B. (1990). Evaluating teachers for career awards and merit pay. En J. Millman \& L. Darling-Hammond (Eds.). The New Handbook of Teacher Evaluation. Assessing Elementary and Secondary School Teachers (pp.133-146). California: Sage Publications.

Barber, L. W. (1990). Self-assessment. En J. Millman \& L. Darling-Hammond (Eds.). The New Handbook of Teacher Evaluation. Assessing Elementary and Secondary School Teachers (pp.216-228). California: Sage Publications.

Barr, A. S., \& Emans, L. M. (1930). What qualities are prerequisite to success in teaching? Nation's Schools, 6, 60-64.

Boyan, N., \& Copeland, W. (1978). Instructional supervision training program. Columbus, Oh: Charles E. Merril.

Carroll, J. G. (1981). Faculty self-assessment. En J. Millman (Ed.). Handbook of Teacher Evaluation (pp.180-200). Beverly Hills, Ca: Sage Publications.

De La Orden, A. (1990). Evaluación, Selección y Promoción del profesorado universitario. Revista Complutense de Educación, 1(1), 11-29. Recuperado de https://revistas. ucm.es/index.php/RCED/article/view/RCED9090130011A/18245

De Miguel, M. (1991). Criterios para la evaluación del profesorado universitario. Oviedo: KRK.

De Miguel, M. (1998). La evaluación del profesorado universitario. Criterios y propuestas para mejorar la función docente. Revista de Educación, 315, 67-84. Recuperado de https://dialnet.unirioja.es/servlet/articulo?codigo $=19192$

Denham, C. H. (1987). A perspective on the major purposes and basic procedures for teacher evaluation. Journal of Personnel Evaluation in Education, 1(1), 29-32. Recuperado de https://link.springer.com/article/10.1007/BF00143277

Dwyer, C. A. (1994). Criteria for performance-based teacher assessments: Validity, standards, and issues. Journal of Personnel Evaluation in Education, 8(2), 135-150. Recuperado de https://link.springer.com/article/10.1007/BF00972260

Epstein, J. L. (1985). A question of merit: Principals' and parents' evaluations of teachers. Educational Researchers, 14(7), 3-8. Recuperado de http://journals.sagepub.com/doi/ abs/10.3102/0013189X014007003

Escudero, T. (1980). ¿Se pueden evaluar los centros educativos y sus profesores? Zaragoza: ICE Universidad de Zaragoza

Escudero, T. (1989). Aproximación pragmática a la evaluación de la universidad. Revista Investigación Educativa, 7(13), 93-112. Recuperado de http://revistas.um.es/rie/article/ view/136611/124211

Escudero, T. (1999). Los estudiantes como evaluadores de la docencia y de los profesores: nuestra experiencia. Revista Interuniversitaria de Formación del Profesorado, 34, 69-86. Recuperado de http://www3.uva.es/aufop/publica/revaufop/rev99-34.htm\#Escudero 
Escudero, T (2000). La voz de los estudiantes: un delicado instrumento de evaluación, Cuadernos IRC, 5, 31-38.

Escudero, T. (2003). Desde los tests hasta la investigación evaluativa actual. Un siglo, el $\mathrm{XX}$, de intenso desarrollo de la evaluación en educación. RELIEVE-Revista Electrónica de Investigación y Evaluación Educativa, 9(1), 1-33 Recuperado de https://www.uv.es/ RELIEVE/v9n1/RELIEVEv9n1_1.htm

Escudero, T. (2005). Selección, evaluación y carrera profesional. Ponencia presentado en I foro ANECA "El profesorado universitario", Madrid: ANECA

Escudero, T. (2005-2006). Claves identificativas de la investigación evaluativa: Análisis desde la práctica. Contextos Educativos, 8-9, 179-199. Recuperado de https://publicaciones.unirioja.es/ojs/index.php/contextos/article/view/563

Escudero, T. (2006a). Evaluación y mejora de la calidad en educación, en T. Escudero \& A.D. Correa (Ed.). Investigación en innovación educativa algunos ámbitos relevantes (pp. 269-325). Madrid: Editorial La Muralla.

Escudero, T. (junio, 2006b). La evaluación del profesorado en España en la última década: Nuevos retos. Ponencia presentada en Encuentro 10 años de evaluación de la calidad de las universidades españolas: 1996-2006 Resultados y objetivos (ANECAUniversidad de Zaragoza), Jaca.

Escudero, T. (2010). Fundamentos de la investigación evaluativa en educación, en S. Nieto (Ed.). Principios, Métodos y Técnicas esenciales para la Investigación Educativa (pp. 497-523), Madrid: Dykinson.

Escudero, T. (2011). La construcción de la investigación evaluativa. El aporte desde la educación. Zaragoza: Universidad de Zaragoza

Escudero, T. (2016a). La investigación evaluativa en el siglo XXI: un instrumento para el desarrollo educativo y social cada vez más relevante. RELIEVE-Revista Electrónica de Investigación y Evaluación Educativa, 22(1), 1-20. doi: https://doi.org/10.7203/ relieve.22.1.8164

Escudero, T. (2016b). Evaluar al Profesorado. Heraldo de Aragón. Recuperado de https:// www.heraldo.es/noticias/heraldo-premium/tribuna/2016/05/22/evaluar-profesorado-873228-2091031.html

Escudero, T. (2018). La selección y promoción del profesorado universitario Heraldo de Aragón, Recuperado de https://www.heraldo.es/index.php/mod.global/mem. buscadorHemeroteca

Escudero, T., Pino, J. L., \& Rodríguez, C. (2010). Evaluación del profesorado universitario para incentivos individuales: revisión metaevaluativa. Revista de Educación, 351, 513-537. Recuperado de https://www.mecd.gob.es/revista-de-educacion/numerosrevista-educacion/numeros-anteriores/2010/re351/re351_21.html

Fernández, M. M., Navarro, C., Higuera, C., \& Martínez, E. (1993). Encuesta al profesorado de primaria y secundaria de la enseñanza pública. Madrid: CIDE.

Frase, L. E., \& Streshly, W. (1994). Lack of accuracy, feedback, and, commitment in teacher evaluation. Journal of Personnel Evaluation in Education, 8(1), 47-57. Recuperado de https://link.springer.com/article/10.1007/BF00972709

García Ramos, J. M. (1997). Valoración de la competencia docente del profesor universitario. Revista Complutense de Educación, 8(2), 81-108. Recuperado de http://revistas. ucm.es/index.php/RCED/article/view/RCED9797220081A 
Gellman, E. S., \& Berkowitz, M. (1992). Factors perceived as important in teacher evaluation, The Alberta Journal of Educational Research, 38(3), 219-234.

Genova, W. J. (1976). Mutual Benefit Evaluation of Faculty and Administrators in Higher Education. Cambridge, Ma: Ballinger

Glass, G. V. (1990). Using student test scores to evaluate teachers, en J. Millman \& L. Darling-Hammond (Eds.). The New Handbook of Teacher Evaluation. Assessing Elementary and Secondary School Teachers (pp. 229-240), California: Sage Publications.

González, M. C. (1992). La evaluación de profesores, Proyecto de Investigación. Madrid: CIDE. Haney, W. (1990). Preservice evaluation of teachers, en J. Millman \& L. DarlingHammond (Eds.). The New Handbook of Teacher Evaluation. Assessing Elementary and Secondary School Teachers (pp.46-61). California: Sage Publications.

Harris, B. M. (1986). Developmental teacher evaluation. Boston: Allyn \& Bacon,

Hatry, H. P., \& Greiner, J. M. (1985). Issues and case studies in teacher incentive plans. Washington, D.C: The Urban Institute Press.

Joint Committee on Standards for Educational Evaluation (1981). Standards for evaluations of educational programs, projects and materials. New York: McGraw-Hill

Joint Committee on Standards for Educational Evaluation (1988). The personnel evaluation standards: How to assess systems for evaluating educators. California: Sage Publications

Jornet, J. M. (2017). Editorial. Revista de Investigación Educativa, 35(2), 299-306. Recuperado de http://revistas.um.es/rie/article/view/299021/214071

Klenowski, V. (2007). Desarrollo del Portafolios para el Aprendizaje y la Evaluación. Madrid: Narcea.

Malen, B. (1999). On rewards, punishments, and possibilities: Teacher compensation as an instrument for education reform. Journal of Personnel Evaluation in Education, 12(4), 387-394. Recuperado de https://link.springer.com/article/10.1023/A:1008020029508

Marczely, B. (1992). Teacher evaluation: Research versus practice. Journal of Personnel Evaluation in Education, 5(3), 279-290. Recuperado de https://link.springer.com/ article/10.1007/BF00125242

Marsh, H. W., \& Hocevar, D. (1991). The multidimensionality of students' evaluation of teaching effectiveness: The generality of factor structures across academic discipline, instructor level and course level. Teacher $\mathcal{E}$ Teacher Education, 7(1), 9-18. doi: https:// doi.org/10.1016/0742-051X(91)90054-S

Mateo, J. (1993). La evaluación del profesorado de secundaria, Ponencia en el Seminario sobre las re-formas educativas actuales en España. Su evaluación: metodología y resultados. Madrid: CIDE-UNED-CDLM

Mateo, J. Escudero, T., De Miguel, M., Mora, J. G., \& Rodríguez, S. (1995). La evaluación del profesorado de secundaria: la opinión del profesor, Proyecto de Investigación del CIDE, Memoria final. Barcelona: CIDE.

Mateo, J. Escudero, T., De Miguel, M., Mora, J. G., \& Rodríguez, S. (1996a). La evaluación del profesorado de secundaria. Barcelona: Cedecs Editorial

Mateo, J. Escudero, T., De Miguel, M., Mora, J. G., \& Rodríguez, S. (1996b). La evaluación del profesorado. Un tema a debate. Revista de Investigación Educativa, 14(2), 73-93. Recuperado de http://revistas.um.es/rie/article/view/141222/127072 
Mclaughlin, M. W. (1990). Embracing contraries: Implementing and sustaining teacher evaluation, en J. Millman \& L. Darling-Hammond (Eds.). The New Hadbook of Teacher Evaluation. Assessing Elementary and Secondary School Teachers (pp: 403-415), California: Sage Publications.

Mehrens, W. A. (1990). Combining evaluation data from multiple sources, en J. Millman, \& L. Darling-Hammond, (Eds.). The New Handbook of Teacher Evaluation. Assessing Elementary and Secondary School Teachers (pp.322-334), California: Sage Publications

Muñoz Cantero, J.M., Ríos De Deus, M.P., \& Abalde, E. (2002). Evaluación Docente vs. Evaluación de la Calidad. Revista Electrónica de Investigación y Evaluación Educativa (RELIEVE), 8(2), 103-134. doi: https://doi.org/10.7203/relieve.8.2.4362

Muñoz Cantero, J. M, \& Espiñeira, E. M. (2010). Plan de Mejoras fruto de la evaluación de la calidad de la atención a la diversidad en un centro educativo. Revista de Investigación Educativa, 28(2), 245-264. Recuperado de http://revistas.um.es/rie/ article/view/111931/121571

Murphy, J. (1987). Teacher evaluation: A comprehensive framework for supervisor. Journal of Personnel Evaluation in Education, 1(2), 157-180. Recuperado de https:// link.springer.com/article/10.1007\%2FBF00128892

Natriello, G. (1990). Intended and unintendend consequences: Purposes and effects of teacher evaluation. En J. Millman, \& L. Darling-Hammond, (Eds.). The New Handbook of Teacher Evaluation. Assessing Elementary and Secondary School Teachers (pp. 35-45). California: Sage Publications

Nevo, D. (1994). How can teachers benefit from teacher evaluation?. Journal of Personnel Evaluation in Education, 8(2), 109-117. Recuperado de https://ink.springer.com/ article/10.1007\%2FBF00972258

Palacián, E., \& Blanco, F. (1993). Profesión docente, reforma y transferencias: Visión del profesorado. Zaragoza: ICE-Universidad de Zaragoza.

Pérez Juste, R., Cristobal, M., \& Martínez, B. (Coords.). (1995). Evaluación de Profesores y Reformas Educativas. Madrid: UNED.

Peterson, K. D. (1990). Assistance and assessment for beginning teachers. En J. Millman, \& L. Darling-Hammond (Eds.). The New Handbook of Teacher Evaluation. Assessing Elementary and Secondary School Teachers (pp.104-115). California: Sage Publications

Peterson, K. D. (1995). Teacher Evaluation. A comprehensive guide to new directions and practices. California: Corwin Press

Peterson, K. D., Stevens, D., \& Mack, C. (2001). Presenting complex teacher evaluation data: Adventages of dossier organization techniques over portfolios. Journal of Personnel Evaluation in Education, 15(2), 121-133. Recuperado de https://link.springer. com/article/10.1023/A:1012443829862

Popham, W. J. (1973). Evaluating instruction. Englewood-NJ: Prentice-Hall

Popham, W. J. (1987). The shortcomings of champagne teacher evaluations. Journal of Personnel Evaluation in Education, 1, 25-28. Recuperado de https://link.springer.com/ article/10.1007\%2FBF00143276

Riegle, R. P. (1987). Conceptions of faculty development. Educational Theory, 37, 53-59. doi: https://doi.org/10.1111/j.1741-5446.1987.00053.x 
Rodriguez Espinar, S. (1997). La evaluación institucional universitaria. Revista de Investigación Educativa, 15(2), 179-216. Recuperado de http://revistas.um.es/rie/article/ view/122631

Rodriguez Espinar, S. (2003). Evaluación comprensiva del profesorado universitario. Educación Médica, 6(3), 25-30. Recuperado de http://scielo.isciii.es/scielo. php?script=sci_arttext\&pid=S1575-18132003000300013

Rosenshine, B. (1970). The stability of teacher effects upon student achievement. Review of Educational Research, 40, 647-662. Recuperado de https://www.jstor.org/stable/1169461

Scriven, M. (1981). Summative teacher evaluation, en J. Millman (Ed.), Hankbook on teacher evaluation (pp. 244-271), Beverly Hills-California: Sage Publications

Scriven, M. (1988). Duty-based teacher evaluation. Journal of Personnel Evaluation in Education, 1(4), 319-334. Recuperado de https://link.springer.com/ article/10.1007\%2FBF00124098

Scriven, M. (1990). Teacher selection. En J. Millman \& L. Darling-Hammond (Eds.). The New Handbook of Teacher Evaluation. Assessing Elementary and Secondary School Teachers (pp. 76-103), Newbury Park-California: Sage Publications, Newbury Park

Scriven, M. (1994). Duties of the teacher. Journal of Personnel Evaluation in Education, 8(2), 151-184. Recuperado de https://link.springer.com/article/10.1007\%2FBF00972261

Scriven, M., (1995). A unified theory approach to teacher evaluation. Studies in Educational Evaluation, 21(2), 111-129. doi: https://doi.org/10.1016/0191-491X(95)00009-J

Schalock, M. D., Cowart, B., \& Staebler, B. (1993). Teacher productivity revisited: Definition, theory, measurement and application. Journal of Personnel Evaluation in Education, 7(2), 179-196. Recuperado de https://link.springer.com/article/10.1007\%2FBF00995302

Seldin, P. (1997). The teaching portfolio: A practical guide to improved performance and promotion/tenure decisions. Bolton, Ma.: Anker.

Shinkfield, A. J., \& Stufflebeam, D. L. (1997). Teacher evaluation: Guide to effective practice. Boston, Ma.: Kluwer Academic Publishers

Stiggins, R. J., \& Bridgeford, N. J. (1985). Performance assessment for teacher development, Educational Evaluation and Policy Analysis, 7(1), 85-97. doi: https://doi. org/10.3102/01623737007001085

Stodolsky, S. S. (1990). Classroom Observation. En J. Millman \& L. Darling-Hammond (Eds.). The New Handbook of Teacher Evaluation. Assessing Elementary and Secondary School Teachers (pp. 175-190), Newbury Park, California: Sage Publications

Stufflebeam, D. L. (1991). An introduction to the Center for Research on Education Accountability and Teacher Evaluation (CREATE). Journal for Personnel Evaluation in Education, 5(1), 85-94.

Stufflebeam, D. L., \& Brethower, D. M. (1987). Improving personnel evaluations through pro-fessional standards. Journal of Personnel Evaluation in Education, 1, 125-155. Recuperado de https://link.springer.com/article/10.1007\%2FBF00128891

Stufflebeam, D. L., \& Shinkfiel, A. J. (1987). Evaluación sistemática. Guía teórica y práctica. Madrid: Ediciones Paidós/M.E.C.

Stufflebeam, D. L., \& Sanders, J. R. (1990). Using the Personnel Evaluation Standards to Improve Teacher Evaluation, en J. Millman \& L. Darling-Hammond (Eds.). The New Handbook of Teacher Evaluation. Assessing Elementary and Secondary School Teachers (pp. 416-428), Newbury Park, California: Sage Publications 
Tejedor, F. J. (2003). Un modelo de evaluación del profesorado universitario, Revista de Investigación Educativa, 21(1), 157-182. Recuperado de http://revistas.um.es/rie/ article/view/99151

Tejedor, F.J., \& Jornet, J. M. (2008). La evaluación del profesorado universitario en España. Revista Electrónica de Investigación Educativa, 10(Especial), 1-29 Recuperado de http:// www.scielo.org.mx/scielo.php?script=sci_arttext\&pid=S1607-40412008000300005

Veenman, S. (1984). Perceived problems of beginning teachers. Review of Educational Research, 54, 143-178. Doi https://doi.org/10.3102/00346543054002143

Villa, A. (1985). La evaluación del profesor: perspectivas y resultados. Revista de Educación, 277, 55-93. Recuperado de https://www.mecd.gob.es/revista-de-educacion/ numeros-revista-educacion/numeros-anteriores/1985/re277/re277_04.html

Weiss, C. H. (1998). Evaluation. Methods for studying programs and policies. Upper Saddle River, New Jersey: Prentice Hall

Wilson, B., \& Wood, J.A. (1996). Teacher evaluation: A national dilemma. Journal of Personnel Evaluation in Education, 10(1), 75-82. Recuperado de https://link.springer. com/article/10.1007\%2FBF00139470

Wise, A., Darling-Hammond, L. McLaughlin, M., \& Bernstein, H. (1985). Teacher evaluation: A study of effective practices. Elementary School Journal, 86, 61-121. doi: https://doi.org/10.1086/461437

Wolfe, P. (1987). What the «seven-step lesson plan» isn't. Educational Leadership, 45(5), 70-71. Recuperado de http://www.ascd.org/ASCD/pdf/journals/ed_lead/el_198702_ wolfe.pdf

Fecha de recepción: 18 de septiembre de 2018.

Fecha de revisión: 19 de septiembre de 2018.

Fecha de aceptación: 8 de octubre de 2018. 
